\title{
Population Pharmacokinetics of AZD- 5847 in Adults with Pulmonary Tuberculosis
}

\author{
Abdullah Alsultan, a,b Jennifer J. Furin, ${ }^{c}$ Jeannine Du Bois, ${ }^{d}$ Elana van Brakel, ${ }^{d}$ \\ Phalkun Chheng, ${ }^{c}$ Amour Venter, ${ }^{e}$ Bonnie Thiel,c Sara A. Debanne, ${ }^{f}$ \\ W. Henry Boom, ${ }^{c}$ Andreas H. Diacon, ${ }^{\text {d,e }}$ John L. Johnson, ${ }^{c}$ Charles A. Peloquin ${ }^{b}$ \\ King Saud University College of Pharmacy, Riyadh, Saudi Arabiaa; University of Florida College of Pharmacy, \\ Gainesville, Florida, USA \\ Hospitals Cleveland Medical Center, Cleveland, Ohio, USAc; TASK Applied Science, Cape Town, South Africad; \\ MRC Centre for Tuberculosis Research, Tygerberg, South Africae; Department of Population and Quantitative \\ Health Sciences, Case Western Reserve University, Cleveland, Ohio, USAf
}

ABSTRACT AZD-5847 is a new oxazolidinone derivative under development for the treatment of tuberculosis (TB). Here we describe the population pharmacokinetics (PK) of AZD-5847 in patients with tuberculosis based on a recently completed phase II study. The study included 60 patients with drug-susceptible TB. Patients were randomized to four dosages (500 mg once daily, 1,200 mg once daily, $500 \mathrm{mg}$ twice daily, and $800 \mathrm{mg}$ twice daily). Patients were intensively sampled on days 1 and 14 . AZD-5847 pharmacokinetics were best described with a two-compartment model with lag time $\left(T_{\text {lag }}\right)$ for absorption. AZD-5847 bioavailability was nonlinear and plateaued at $800 \mathrm{mg}$. We performed deterministic simulation to compare the PK/pharmacodynamics (PD) of AZD-5847, linezolid, and sutezolid. AZD-5847 PK/PD in terms of both area under the concentration-time curve for the free, unbound fraction (fAUC)/MIC and time the free concentration was above the MIC ( $f \mathrm{~T}>\mathrm{MIC}$ ) were less favorable than those for linezolid and sutezolid. This could help explain the poor bactericidal activity of AZD-5847 in the recent phase II study.

KEYWORDS pharmacokinetics, AZD-5847, tuberculosis -here is an urgent and clear need to improve and shorten the duration of treatment for tuberculosis (TB). One approach to meeting this need is to develop new drugs for TB. Several oxazolidinones are currently under clinical and experimental investigation for the treatment of TB. Linezolid has in vitro and in vivo activities against TB and has been used for the treatment of multidrug-resistant/extremely drug-resistant TB (MDR/XDR-TB) for the last decade. The main concerns with the use of linezolid are neurologic and hematologic toxicity with chronic use. Sutezolid (formerly PNU-100480) is a new oxazolidinone in clinical development for TB treatment. In vitro, animal, and whole-blood bactericidal and early bactericidal activity studies with patients with TB demonstrate that the activity of sutezolid is superior to that of linezolid $(1,2)$. Also, sutezolid seems to have less hematologic toxicity than linezolid (3). AZD-5847 is another oxazolidinone derivative under development for the treatment of TB. The drug recently completed a phase lla clinical trial study to evaluate its pharmacokinetics (PK) and early bactericidal activity at several doses and schedules in patients with drugsusceptible TB (4). The early bactericidal activity of AZD-5847 was very modest in that study. The objective of this work was to develop a population PK model for AZD-5847 based upon patients with TB from the recent phase II study. Such a model could be used for further dose optimization of AZD-5847.
Received 22 May 2017 Returned for modification 24 June 2017 Accepted 14 July 2017

Accepted manuscript posted online 24 July 2017

Citation Alsultan A, Furin JJ, Du Bois J, van Brakel E, Chheng P, Venter A, Thiel B, Debanne SA, Boom WH, Diacon AH, Johnson JL, Peloquin CA. 2017. Population pharmacokinetics of AZD-5847 in adults with pulmonary tuberculosis. Antimicrob Agents Chemother 61:e01066-17. https://doi.org/10 .1128/AAC.01066-17.

Copyright $\odot 2017$ American Society for Microbiology. All Rights Reserved. Address correspondence to Charles A. Peloquin, peloquin@cop.ufl.edu. 
TABLE 1 Baseline demographics

\begin{tabular}{ll}
\hline Characteristic & Value \\
\hline Age (yrs), mean (SD) & $35(10)$ \\
Wt (kg), mean (SD) & $53(8)$ \\
Sex (male), no. (\%) & $50(83)$ \\
HIV negative, no. (\%) & $58(97)$ \\
Bilateral disease on chest radiograph, no. (\%) & $23(38)$ \\
Cavitary disease on chest radiograph, no. (\%) & $57(95)$ \\
\hline
\end{tabular}

\section{RESULTS}

A total of 1,723 samples were used for the analysis. The average age in the population was 35 years. The average weight was $53 \mathrm{~kg}$. A total of $83 \%$ of the participants were male, and $97 \%$ were HIV negative (Table 1).

Population pharmacokinetics. A two-compartment model with linear elimination and $\left(T_{\text {lag }}\right)$ for absorption adequately described the data. AZD-5847 showed nonlinear absorption, likely because of saturable absorption. Initially we modeled the data for day 1 only. The population pharmacokinetic model for day 1 was used to predict the concentrations on day 14. As shown in Fig. 1, there was no evidence of any timedependent changes in clearance $(C L)$ /bioavailability $(F)$ or bioavailability. Data sets for both day 1 and day 14 then were combined into one data set and modeled. Parameter estimates for the final model are shown in Table 2. Bioavailability started to decrease above the $800-\mathrm{mg}$ dose and was $67 \%$ at the 1,200-mg dose. The bioavailability of the $800-\mathrm{mg}$ dose was similar to that in subjects receiving $500 \mathrm{mg}$. Therefore, patients were categorized into two groups: those receiving $500 \mathrm{mg}$ and $800 \mathrm{mg}$ were considered group $A$ and those receiving 1,200 mg were considered group B. Figure 2 shows the typical PK profile for AZD-5847 at steady state. No significant covariates were identified in the analysis. Goodness-of-fit plots are shown in Fig. 3 and 4.

Simulations. We performed deterministic simulations to compare the PK/pharmacodynamics (PD) of different oxazolidinones. We estimated area under the concentrationtime curve for the free, unbound fraction from 0 to $24 \mathrm{~h}\left(\mathrm{fAUC}_{\mathrm{O}-24}\right)$ at steady state for AZD-5847, linezolid, and sutezolid and its active metabolite following standard dosing regimens (Table 3). Linezolid at $600 \mathrm{mg}$ twice daily (BID) achieved the highest fAUC/
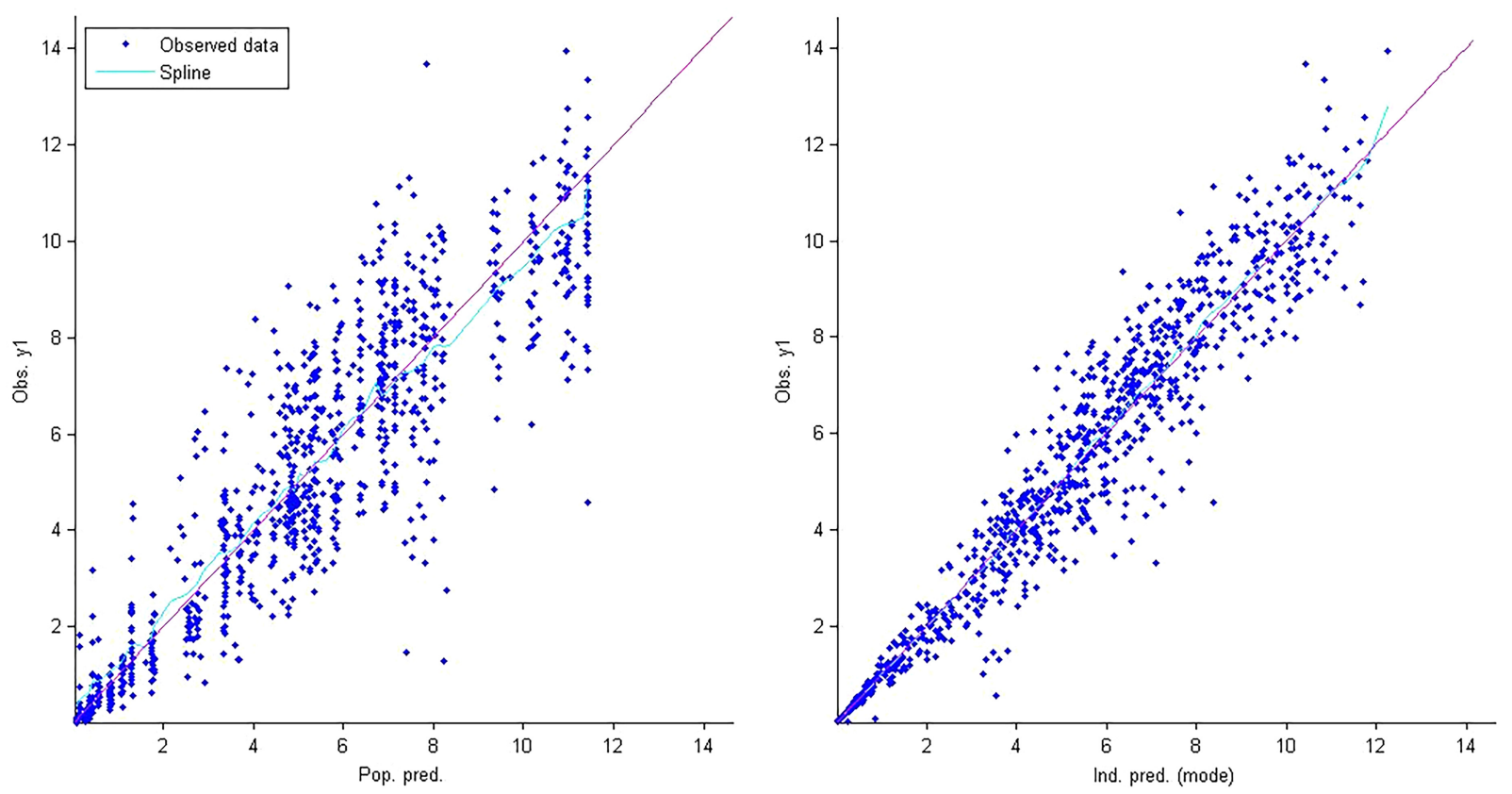

FIG 1 Population pharmacokinetic model for day 1 only used to predict concentrations on day 14 . 
TABLE 2 Pharmacokinetic parameter estimates for the final population model ${ }^{a}$

\begin{tabular}{ll}
\hline PK parameter & Estimate (\% RSE) \\
\hline$F \quad$ Group A & 1 (fixed) \\
Group B & $0.65(3)$ \\
$T_{\text {lag }}(\mathrm{h})$ & \\
$\%$ IIV for $T_{\text {lag }}(\% \mathrm{CV})$ & $0.3(13)$ \\
$K_{a}\left(\mathrm{~h}^{-1}\right)$ & $60(16)$ \\
$\%$ IIV for $K_{a}(\% \mathrm{CV})$ & $0.439(6)$ \\
$V_{1}$ (liters) & $21.5(16)$ \\
$\%$ IIV for $V_{1}(\% \mathrm{CV})$ & $48.2(8)$ \\
$\mathrm{CL}$ (liters $/ \mathrm{h})$ & $15.6(19)$ \\
$\%$ IIV for CL $(\% \mathrm{CV})$ & $7.94(3)$ \\
$V_{2}$ (liters) & $21.9(10)$ \\
$\%$ IIV for $V_{2}(\% \mathrm{CV})$ & $28.9(14)$ \\
$\mathrm{Q}$ (liters/h) & $62.6(18)$ \\
$\%$ IIV for Q $(\% \mathrm{CV})$ & $8.74(18)$ \\
$\mathrm{b}$ & $54.3(15)$ \\
\hline
\end{tabular}

alIV, interindividual variability; $\mathrm{CV}$, coefficient of variation; $K_{a}$ absorption rate constant; $V_{1}$, volume of distribution in the central compartment; $V_{2}$, volume of distribution for the peripheral compartment; $Q$, intercompartmental clearance; $b$, proportional error.

MIC, followed by sutezolid at $600 \mathrm{mg}$ BID or 1,200 once daily (QD), linezolid at $600 \mathrm{mg}$, and AZD-5847 at both 500 and $800 \mathrm{mg} \mathrm{BID}$; the sutezolid metabolite showed the lowest $F A \cup C / M I C$. If we combined the $F A \cup C$ for sutezolid and its active metabolite, this drug would have an $f A U C / M I C$ similar to that of linezolid at $600 \mathrm{mg}$ BID. The times the free concentration was above the MIC ( $f \mathrm{~T}>\mathrm{MIC}$ ) were similar for linezolid and AZD$5847,100 \%$ for BID dosing and approximately 50\% for QD dosing. We did not estimate the $f \mathrm{~T}>\mathrm{MIC}$ for sutezolid and its metabolite, as no published population pharmacokinetic model was identified.

\section{DISCUSSION}

AZD-5847 shows biphasic elimination. Absorption of AZD-5847 is nonlinear, and administering doses above $800 \mathrm{mg}$ might not be beneficial. In the mouse model, a minimum $f A U C / M I C$ of $>20$ was required for bactericidal activity. In the phase II study, the median $f A U C / M I C$ was $<18$ for all four dosing regimens. This could help explain the poor bactericidal activity observed in the phase II study.

Our simulations show that AZD-5847 has less favorable PK/PD than those of the other oxazolinones. The fAUC/MIC was 3-fold higher for linezolid at $600 \mathrm{mg}$ BID than for AZD-5847 at $800 \mathrm{mg} \mathrm{BID}$. The $f A U C / M I C$ was also higher for the sutezolid at 1,200 mg QD than for AZD-5847 at $800 \mathrm{mg}$ BID. In a study by Zhang et al., AZD-5847 showed no bactericidal activity against nonreplicating bacilli in a mouse model despite being active in vitro (5). In contrast, sutezolid showed potent activity in vivo against nonrep-

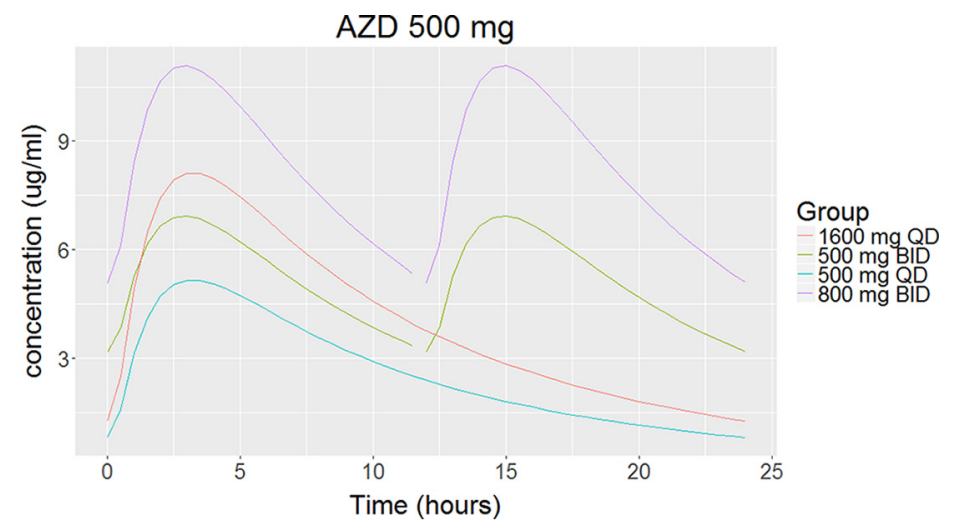

FIG 2 Typical pharmacokinetic profile for AZD-5847. 

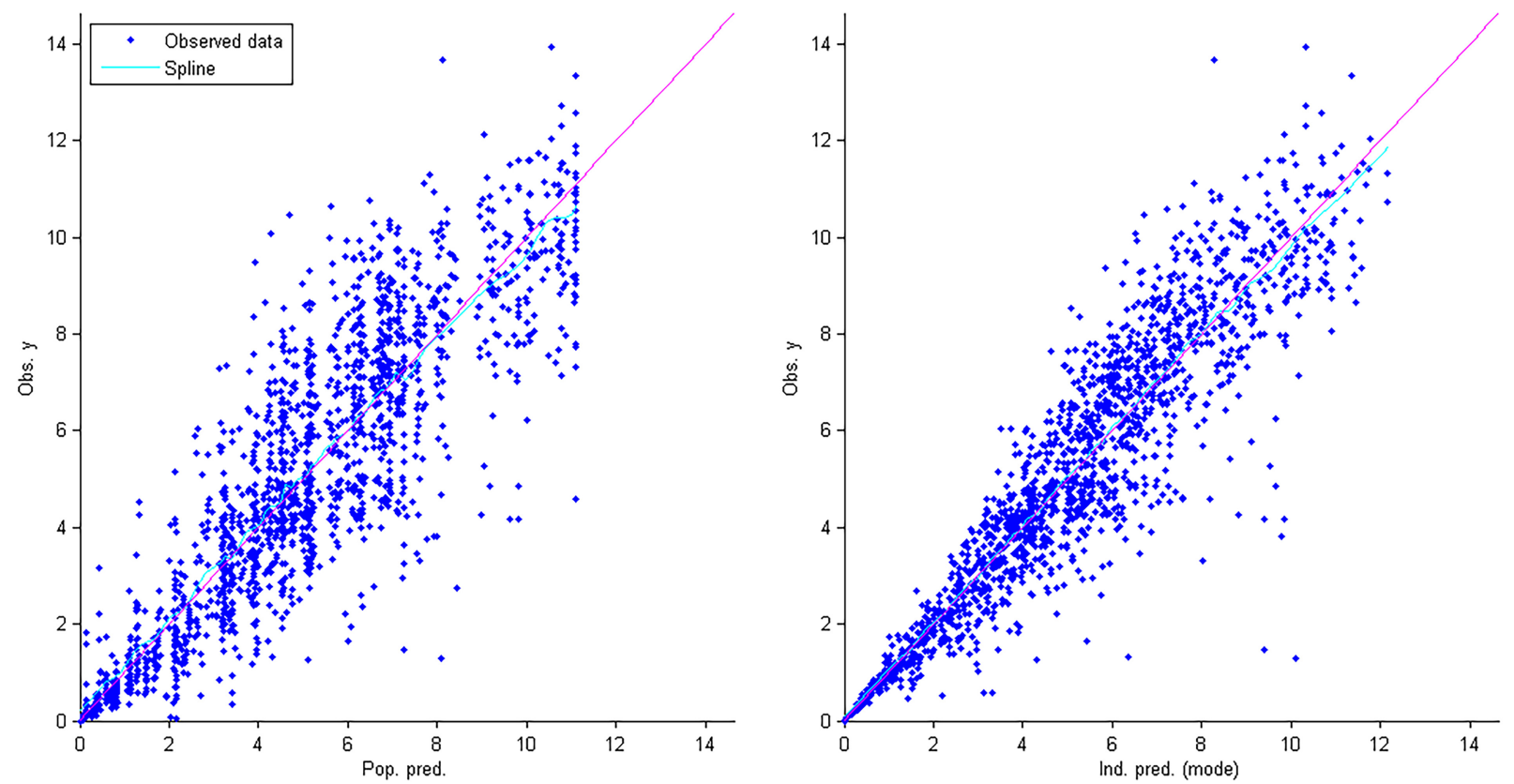

FIG 3 Goodness-of-fit plots for the final population pharmacokinetic model. The graph on the right shows individual predictions of AZD-5847 versus observed concentrations. The graph on the left shows population predictions of vancomycin versus observed concentrations.

licating bacilli in the mouse model. A possible explanation for the poor activity observed for AZD-5847 in vivo is the high level of protein binding. High protein binding limits the drug's activity and may limit penetration into cavitary lesions. Increasing the dose of AZD-5847 could improve its bactericidal activity. However, given the saturable absorption of AZD-5847, it is difficult to achieve favorable PK/PD targets. Also, given the adverse effects observed the phase II study, it might be difficult to administer AZD-5847 more frequently.
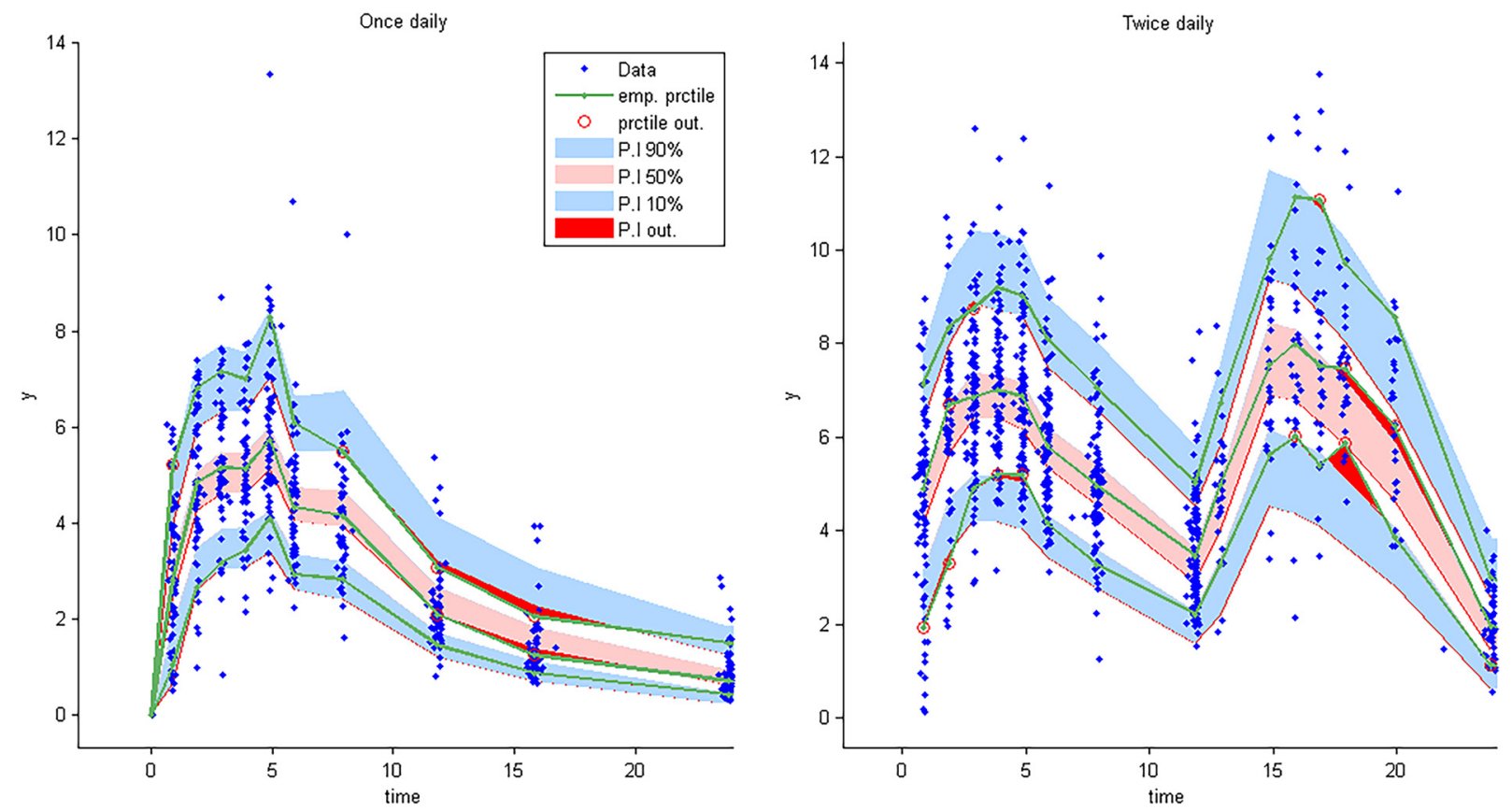

FIG 4 Visual predictive check for AZD-5847 concentration versus time based on 1,000 Monte Carlo simulations. The solid green line represents the 10th, 50th, and 90th percentiles of observed data. The shaded regions represent the $90 \%$ confidence interval around the 10 th, 50th, and 90 th percentiles of simulated data. The blue diamonds are observed concentrations. 
TABLE 3 PK/PD for AZD-5847, linezolid, and sutezolid and its active metabolite

\begin{tabular}{llll}
\hline Drug & AUC $_{\mathbf{0 - 2 4}}(\boldsymbol{\mu g} \cdot \mathbf{h} / \mathrm{ml})$ & fAUC/MIC & fT $>$ MIC (\%) \\
\hline Linezolid & & & \\
$600 \mathrm{mg}$ QD & 84.5 & 59 & 58.3 \\
$600 \mathrm{mg} \mathrm{BID}$ & 169 & 118 & 100 \\
& & & \\
AZD-5847 & 126 & 25 & 50 \\
$500 \mathrm{mg}$ BID & 201 & 40 & 100 \\
$800 \mathrm{mg}$ BID & & & \\
Sutezolid & 7 & 59 & $\mathrm{NA}$ \\
$600 \mathrm{mg}$ BID & 7 & 59 & $\mathrm{NA}$ \\
$1200 \mathrm{mg}$ QD & 39 & 39 & $\mathrm{NA}$ \\
& 36 & 36 & $\mathrm{NA}$ \\
\hline
\end{tabular}

aNA, not available.

\section{MATERIALS AND METHODS}

Study design. Study participants included HIV-uninfected and -infected men and women aged 18 to 65 years from the Western Cape province of South Africa with initial episodes of newly diagnosed, sputum smear-positive, drug-susceptible (susceptible to isoniazid and rifampin) pulmonary tuberculosis and reasonably normal renal and hepatic function. Patients with diabetes mellitus were excluded. Patients with HIV infection were eligible if they had a CD4 cell count of more than $350 / \mu \mathrm{l}$ and there was no indication to start antiretroviral therapy during the course of the study. Patients with suspected miliary or meningeal TB who required immediate combination anti-TB treatment were excluded. Most patients in the study were heavily smear positive, had cavitary disease, and had a high initial sputum bacillary load (4). Patients were randomized to four treatment arms as follows: $500 \mathrm{mg}$ once daily, 500 mg twice daily, $800 \mathrm{mg}$ twice daily, and 1,200 mg once daily. PK sampling occurred on days 1 and 14 . Blood samples were collected at $0,1,2,3,4,5,6,8,12,13,15,16,17,18,20$, and $24 \mathrm{~h}$.

Analytical assay. Concentrations of AZD5847 and its carboxylic acid metabolite in plasma were measured using a validated high-performance liquid chromatography (HPLC) assay. A Thermo Electron SpectraSYSTEM with photodiode array (PDA) detection was used. Parent standard concentrations ranged from 0.010 to $5.0 \mu \mathrm{g} / \mathrm{ml}$, and the standard curves were fitted with $1 / \mathrm{y} 2$ weighted linear regression. Overall assay precision was 3 to $18 \%$ across the range.

Population pharmacokinetics. All data were analyzed with Monolix 4.3 using the SAEM algorithm (6). For the structural model, we evaluated one- and two-compartment systems with linear and nonlinear elimination. The absorption was tested with first-order absorption with or without $\left(T_{\text {lag }}\right)$, zero-order absorption, and gastrointestinal (Gl) transit compartment. In the noncompartmental analysis, we noticed that AZD-5847 had saturable absorption. Patients receiving 1,200 mg QD had less than a proportional increase in AUC compared to those receiving $500 \mathrm{mg}$ QD. Therefore, bioavailability $(F)$ was added as a term in the model to evaluate the effect of dose on bioavailability. Dose effect was modeled as a categorical covariate. Bioavailability was fixed at 1 for the 500-mg group and was separately estimated for the 800- and 1,200-mg doses.

First, we started by modeling data for day 1 only. Then the model developed for day 1 was used to predict concentrations on day 14 to assess if there were any time-dependent changes in $\mathrm{CL} / \mathrm{F}$ or bioavailability. In the final step, data for day 1 and 14 were combined in one data set and modeled simultaneously. Pharmacokinetic parameters were assumed to follow a lognormal distribution. A proportional error was used to describe the residual variability. For covariate testing, we started by plotting the empirical Bayesian estimates versus covariates to identify possible correlations. Potentially significant covariates were tested in a stepwise fashion. A $P$ value of 0.1 was included in the model, and a $P$ value of 0.05 was retained in the model. Covariates tested included age, weight, sex, body mass index (BMI), HIV coinfection status, and serum albumin, bilirubin, aspartate transaminase (AST), and alanine transaminase (ALT). Throughout the analysis, model selection was guided by the goodness-of-fit plots, objective function value, and precision of the parameter estimates.

Simulations. Deterministic simulations were performed to estimate $\mathrm{AAUC}_{0-24} / \mathrm{MIC}$ and the time the free concentration was above MIC ( $f \mathrm{~T}>\mathrm{MIC}$ ) for different oxazolidinones. For sutezolid and its active metabolite, AUC was obtained from a previously published noncompartmental analysis (2). $f \mathrm{~T}>\mathrm{MIC}$ was not estimated for sutezolid or its active metabolite, as no published population pharmacokinetic study was identified. For linezolid and AZD-5847, AUC was calculated from the CL/F estimate as follows: $\mathrm{AUC}_{0-24}=D / C \mathrm{~L}$, where $D$ is the dose. To estimate the $\mathrm{fT}>\mathrm{MIC}$ for AZD-5847 and linezolid, concentrations were simulated at 30-min intervals. Then we estimated the $\mathrm{F} \% \mathrm{~T}>\mathrm{MIC}$. The previously published pharmacokinetic model by McGee et al. was used to estimate the linezolid AUC and concentrations (7). The protein binding levels for AZD-5847, linezolid, sutezolid, and the active metabolite of sutezolid were set at $80 \%, 30 \%, 48 \%$, and $11 \%$ (5). The median MICs for AZD-5847, linezolid, sutezolid, and the active metabolite of sutezolid were set at $1 \mu \mathrm{g} / \mathrm{ml}, 0.5 \mu \mathrm{g} / \mathrm{ml}, 0.062 \mu \mathrm{g} / \mathrm{ml}$, and $0.5 \mu \mathrm{g} / \mathrm{ml}(2,4,8)$. 


\section{ACKNOWLEDGMENT}

Abdullah Alsultan is very grateful to the Deanship of Scientific Research and Research

Center, College of Pharmacy, King Saud University, Riyadh, Saudi Arabia.

\section{REFERENCES}

1. Egelund EF, Alsultan A, Peloquin CA. 2015. Optimizing the clinical pharmacology of tuberculosis medications. Clin Pharmacol Ther 98:387-393. https://doi.org/10.1002/cpt.180.

2. Wallis RS, Dawson R, Friedrich SO, Venter A, Paige D, Zhu T, Silvia A, Gobey J, Ellery C, Zhang Y, Eisenach K, Miller P, Diacon AH. 2014. Mycobactericidal activity of sutezolid (PNU-100480) in sputum (EBA) and blood (WBA) of patients with pulmonary tuberculosis. PLoS One 9:e94462. https://doi .org/10.1371/journal.pone.0094462.

3. Wallis RS, Jakubiec W, Kumar V, Bedarida G, Silvia A, Paige D, Zhu T, Mitton-Fry M, Ladutko L, Campbell S, Miller PF. 2011. Biomarker-assisted dose selection for safety and efficacy in early development of PNU100480 for tuberculosis. Antimicrob Agents Chemother 55:567-574. https://doi.org/10.1128/AAC.01179-10.

4. Furin JJ, Du Bois J, van Brakel E, Chheng $P$, Venter A, Peloquin CA, Alsultan A, Thiel BA, Debanne SM, Boom WH, Diacon AH, Johnson JL. 2016. Early bactericidal activity of AZD5847 in patients with pulmonary tuberculosis. Antimicrob Agents Chemother 60:6591-6599. https://doi.org/10.1128/ AAC.01163-16.

5. Zhang M, Sala C, Dhar N, Vocat A, Sambandamurthy VK, Sharma S,
Marriner G, Balasubramanian V, Cole ST. 2014. In vitro and in vivo activities of three oxazolidinones against nonreplicating Mycobacterium tuberculosis. Antimicrob Agents Chemother 58:3217-3223. https://doi.org/ 10.1128/AAC.02410-14.

6. Lavielle M, Mentre F. 2007. Estimation of population pharmacokinetic parameters of saquinavir in HIV patients with the MONOLIX software. Pharmacokinet Pharmacodyn 34:229-249. https://doi.org/10.1007/ s10928-006-9043-z.

7. McGee B, Dietze R, Hadad DJ, Molino LP, Maciel EL, Boom WH, Palaci M, Johnson JL, Peloquin CA. 2009. Population pharmacokinetics of linezolid in adults with pulmonary tuberculosis. Antimicrob Agents Chemother 53:3981-3984. https://doi.org/10.1128/AAC.01378-08.

8. Alcalá L, Ruiz-Serrano MJ, Pérez-Fernández Turégano C, García De Viedma D, Díaz-Infantes M, Marín-Arriaza M, Bouza E. 2003. In vitro activities of linezolid against clinical isolates of Mycobacterium tuberculosis that are susceptible or resistant to first-line antituberculous drugs. Antimicrob Agents Chemother 47:416-417. https://doi.org/10 .1128/AAC.47.1.416-417.2003. 